\title{
Theory of stochastic transitions in area preserving maps
}

\author{
Massimo Tessarotto $^{1}{ }^{a, c}$ and Piero Nicolini ${ }^{2} a, b$ \\ ${ }^{a}$ Department of Mathematics and Informatics, University of Trieste, Italy \\ ${ }^{b}$ National Institute of Nuclear Physics (INFN) Trieste Section, Italy \\ ${ }^{c}$ Consortium for Magnetofluid Dynamics, University of Trieste, Italy
}

\begin{abstract}
A famous aspect of discrete dynamical systems defined by area-preserving maps is the physical interpretation of stochastic transitions occurring locally which manifest themselves through the destruction of invariant KAM curves and the local or global onset of chaos. Despite numerous previous investigations (see in particular Chirikov [1], Greene [2], Percival [3], Escande and Doveil [4] and MacKay [5]) based on different approaches, several aspects of the phenomenon still escape a complete understanding and a rigorous description. In particular Greene's approach is based on several conjectures, one of which is that the stochastic transition leading to the destruction of the last KAM curve in the standard map is due the linear destabilization of the elliptic points belonging to a peculiar family of invariants sets $\{I(m, n)\}$ (rational iterates) having rational winding numbers and associated to the last KAM curve. Purpose of this work is to analyze the nonlinear phenomena leading to the stochastic transition in the standard map and their effect on the destabilization of the invariant sets associated to the KAM curves, leading, ultimately, to the destruction of the KAM curves themselves.
\end{abstract}

\section{INTRODUCTION: PHENOMENOLOGY OF STOCHASTIC TRANSITIONS}

The phenomenology of stochastic transitions for canonical (and area-preserving) maps, such as the standard map [1], is one of the most popular subjects of investigation in nonlinear dynamics. In particular, in the case of the transition to global chaos this amount to the search of the the so-called last KAM curve, i.e. a continuous invariant curve which separates nearby subdomains of the phase-space $(\Gamma)$ characterized at most only by local chaos. The search of such a KAM curve and the conditions of its possible destruction for arbitrary canonical maps may result potentially a difficult problem. In the past, sufficient conditions for the global stochastic transition, for the standard map and suitable families of area-preserving maps, have been proposed, such as Chirikov Criterion [1]) or Greene's Chaotic Hypothesis [2]. However, despite various attempts (see in particular Chirikov[1], Greene [2], Percival [3], Escande and Doveil [4] and MacKay [5]) based on different approaches, several aspects of the phenomenology of stochastic transitions still escape a complete understanding and a rigorous description.

In two dimensions closed KAM curves can be closed (so-called invariant circles) or open. In the first case they separate phase space since trajectories cannot cross KAM curves, and hence produce the phenomenon of phasespace confinement in phase space. Also open KAM curves, however, can separate space. Discrete invariant sets $J$ do not separate space and therefore cannot give rise to phase-space confinement. In general one expects that the winding number of an arbitrary closed (or open) KAM curve, in particular those which separate space, depend on the stochasticity parameter $K$, i.e.,

$$
\alpha=\alpha(J, K) .
$$

Therefore, a KAM curve can be destroyed for appropriate values of $K$, i.e., when $\alpha(J, K)$ becomes rational. This is called local stochastic transition of the KAM curve $J$. The local destruction of a KAM surface, and consequent disappearance of local phase-space confinement, is also called transition to local chaos. In particular, there may exist a special KAM curve, the so-called last KAM curve, whose destruction permits the existence of phase-space

\footnotetext{
1 email: M.Tessarotto@cmfd.univ.trieste.it

2 email: Piero.Nicolini@cmfd.univ.trieste.it

3 Web site: http://cmfd.univ.trieste.it
} 
trajectories which "connect" all boundaries lines of phase space $\Gamma$, thus destroying "global" phase-space confinement. The stochastic transition of this KAM curve is called as global stochastic transition or transition to global chaos.

\section{A CLASSIFICATION OF AREA-PRESERVING MAPS}

For the purpose of attempting a systematic investigation of area-preserving maps, generated by discrete dynamical systems, a convenient approach is to construct them, in analogy to continuous dynamical systems, in terms of a variational principle. Indeed the same concept of Hamiltonian system can be extended to discrete dynamical systems in such a way to yield, by construction, area-preserving (i.e., conservative) maps. By analogy to continuous theory, discrete Hamiltonian systems can be defined by means of a suitable discrete variational principle expressed by a variational equation of the form $\delta S(\mathbf{x})=0$ and denoted as "discrete modified Hamilton variational principle". As usual, this equation must be satisfied by arbitrary syncronous variations of the form $\delta \mathbf{x}_{i}=\left(\delta \mathbf{q}_{i}, \delta \mathbf{p}_{i}\right)$ (with $\left.i=0, n\right)$, being $\delta \mathbf{x}_{i} \equiv \mathbf{x}_{i}-\mathbf{x}_{1 i}$ and $\mathbf{x}_{i}, \mathbf{x}_{1 i}$ two arbitrary elements of the syncronous functional class

$$
\{\mathbf{x}\}=\left\{\mathbf{x}_{i}: \mathbf{x}_{i}, i \in N, \mathbf{x}_{o}=\mathbf{x}^{A}, \mathbf{x}_{n}=\mathbf{x}^{B}, \mathbf{x}_{i}=\left(\mathbf{q}_{i}, \mathbf{p}_{i}\right) \in \Gamma \subseteq R^{2 g}\right\} .
$$

where $\mathbf{x}_{o}=\mathbf{x}^{A}, \mathbf{x}_{n}=\mathbf{x}^{B}$ are suitable compatible boundary conditions and $\mathbf{q}=\left(q_{1}, . ., q_{g}\right), \mathbf{p}=\left(p_{1}, . ., p_{g}\right)$, being $g$ an integer $g \geq 1$ to be identified with the degree of freedom. A convenient definition of the variational functional is

$$
S(\mathbf{x})=\sum_{i \in 0, n}\left\{\left(\mathbf{q}_{i+1}-\mathbf{q}_{i}\right) \cdot \mathbf{p}_{i+1}-H\left(\mathbf{q}_{i}, \mathbf{p}_{i+1}\right)\right\},
$$

where $H\left(\mathbf{q}_{i}, \mathbf{p}_{i+1}\right)$ denotes a suitably smooth real function, here denotes as discrete Hamiltonian function. The corresponding Eulero-Langrange equations read therefore:

$$
\begin{gathered}
\delta S(\mathbf{x})=\sum_{i \in 0, n} \delta \mathbf{p}_{i+1} \cdot\left[\left(\mathbf{q}_{i+1}-\mathbf{q}_{i}\right)-\frac{\partial}{\partial \mathbf{p}_{i+1}} H\left(\mathbf{q}_{i}, \mathbf{p}_{i+1}\right)\right]+ \\
+\sum_{i \in 0, n} \delta \mathbf{q}_{i} \cdot\left[\left(\mathbf{p}_{i}-\mathbf{p}_{i+1}\right)-\frac{\partial}{\partial \mathbf{q}_{i}} H\left(\mathbf{q}_{i}, \mathbf{p}_{i+1}\right)\right]=0,
\end{gathered}
$$

where the partial derivatives should be meant in the sense

$$
\begin{gathered}
\frac{\partial}{\partial \mathbf{p}_{i+1}} H\left(\mathbf{q}_{i}, \mathbf{p}_{i+1}\right)=\left.\frac{\partial}{\partial \mathbf{p}} H\left(\mathbf{q}_{i}, \mathbf{p}\right)\right|_{\mathbf{p}=\mathbf{p}_{i+1}}, \\
\frac{\partial}{\partial \mathbf{q}_{i}} H\left(\mathbf{q}_{i}, \mathbf{p}_{i+1}\right)=\left.\frac{\partial}{\partial \mathbf{q}} H\left(\mathbf{q}, \mathbf{p}_{i+1}\right)\right|_{\mathbf{q}=\mathbf{q}_{i}} .
\end{gathered}
$$

Hence, they deliver the discrete canonical map $T$

$$
\left\{\begin{array}{c}
\mathbf{q}_{i+1}=\mathbf{q}_{i}+\frac{\partial}{\partial \mathbf{p}_{i+1}} H\left(\mathbf{q}_{i}, \mathbf{p}_{i+1}\right), \\
\mathbf{p}_{i+1}=\mathbf{p}_{i}-\frac{\partial}{\partial \mathbf{q}_{i}} H\left(\mathbf{q}_{i}, \mathbf{p}_{i+1}\right), \\
\mathbf{x}_{o}=\mathbf{x}^{A}, \mathbf{x}_{n}=\mathbf{x}^{B},
\end{array}\right.
$$

(defined for $i=0, n$ ) which can be proven to satisfy the fundamental Poisson brackets:

$$
\begin{gathered}
{\left[\mathbf{q}_{i+1}, \mathbf{p}_{i+1}\right]_{\left(\mathbf{q}_{i}, \mathbf{p}_{i}\right)}=\underline{\underline{\mathbf{1}}},} \\
{\left[\mathbf{q}_{i+1}, \mathbf{q}_{i+1}\right]_{\left(\mathbf{q}_{i}, \mathbf{p}_{i}\right)}=\left[\mathbf{p}_{i+1}, \mathbf{p}_{i+1}\right]_{\left(\mathbf{q}_{i}, \mathbf{p}_{i}\right)}=\underline{\underline{\mathbf{0}}}}
\end{gathered}
$$

This implies, by virtue of the previous definition (2) for the variational functional, that discrete Hamiltonian systems, just like continuous ones, result trivially area-preserving. In particular, this property is satisfied by arbitrary elements of the sequence $\left\{x_{i}, i \in N\right\}_{\mathbf{x}^{A}}$, defining the phase-space trajectory of the discrete canonical system, which is determined by the map $T$, for an arbitrary initial state $\mathbf{x}_{o}=\mathbf{x}^{A} \in \Gamma$ (and letting $\mathbf{x}_{n}=\mathbf{x}^{B}$ ).

Conversely, in the particular case of two-dimensional (i.e., for $g=1$ ), it follows that area-preserving maps $T$ of the form

$$
\mathbf{x}_{i} \equiv\left(q_{i}, p_{i}\right) \rightarrow \mathbf{x}_{i+1} \equiv\left(q_{i+1}, p_{i+1}\right) \equiv T \mathbf{x}_{i},
$$

are necessarily canonical and hence of the type defined above (6). 


\section{Parameter-dependent canonical maps}

In the sequel we shall consider, without loss of generality, parameter-dependent $2 \mathrm{D}$ canonical maps $T$ characterized by a bounded phase space $\Gamma \equiv[-\pi, \pi[\times[0,2 \pi[$ and having a discrete Hamiltonian function of the form $H=H\left(q_{i}, p_{i+1}, K\right)$, being $K$ a real parameter (to be denoted as stochasticity parameter) and $H$ a real analytic function of $K$. Moreover, we shall impose that the discrete Hamiltonian $H$ satisfies the constraint

$$
H\left(q_{i}, p_{i+1}, K=0\right)=H_{0}\left(p_{i+1}\right),
$$

for arbitrary $i=0, n$ and phase-space trajectories, which implies

$$
H\left(q_{i}, p_{i+1}, K\right)=H_{0}\left(p_{i+1}\right)+\sum_{j=1}^{\infty} K^{j} H_{j}\left(q_{i}, p_{i+1}\right) .
$$

An example of 2D maps of this type, which results also a twist map, i.e., satisfies the twist condition

$$
\frac{\partial q_{i+1}}{\partial p_{i+1}} \neq 0
$$

is provided by the so-called standard map [[1],[2]].

$$
\begin{aligned}
q_{i+1} & =q_{i}+p_{i+1} \\
p_{i+1} & =p_{i}+K \sin q_{i} .
\end{aligned}
$$

Since the map is manifestly area-preserving, it results also canonical, the discrete Hamiltonian function being in this case:

$$
H\left(q_{i}, p_{i+1}, K\right)=\frac{1}{2} p_{i+1}^{2}+K \cos q_{i} .
$$

Two-dimensional parameter-dependent area-preserving maps of this type are characterized by discrete and continuos invariant sets (the latter called KAM curves). In particular, for $K=0$ all continuous subsets of $\Gamma$ defined by the equations

$$
p_{i}=p^{A}
$$

are necessarily invariant (KAM curves). For $K>0$ KAM curves can change shape or disappear altogether.

In particular, discrete invariant sets may appear which are characterized generally by a finite number ( $n$ denoted as order of the set) of $m$-periodic invariant points (i.e., points which are returned to themselves after $m$ iterations of the map). Discrete and continuous invariant sets can be distinguished by means of their winding numbers $\alpha\left(\mathbf{x}^{A}\right)$, i.e.:

$$
\alpha\left(\mathbf{x}^{A}\right)=\lim _{k \rightarrow \infty} \frac{\delta^{(k)}\left(\mathbf{x}^{A}\right)}{2 \pi k}
$$

where $\delta^{(k)}\left(\alpha\left(\mathbf{x}^{A}\right)\right.$ is the rotation angle after at the $k$ - th iteration of the map $T$, namely:

$$
\delta^{(k)}\left(\mathbf{x}^{A}\right)=\sum_{j=1, k-1}\left(p_{j+1}-p_{j}\right)
$$

and $x_{j}$ is the $j$ - th image of $\mathbf{x}^{A}$ through the canonical map $T$. As a consequence, it is immediate to prove that discrete invariants sets of order $n$ and periodicity $m$, to be denote by the symbol $I(m, n) \equiv\left\{m, n, K, \mathbf{x}^{A}\right\}$, display necessarily a rational winding number $\alpha\left(\mathbf{x}^{A}\right)=m / n$, whereas KAM curves have necessarily irrational winding numbers. In both cases, one expects generally that winding numbers result function of the stochastic parameter $K$, i.e. that $\alpha=\alpha(J, K)$, where $J$ denotes an arbitrary invariant set. In particular one may expect that a given KAM curve $\gamma$ which exists for $K=0$, and is characterized by an irrational winding number, may become a discrete invariant set in correspondence of a suitable critical values of the stochasticity parameter $K$ the function $\alpha(J, K)$ may become rational. This is called local stochastic transition. A basic conjecture (Greene, 1979 [2]) is that for arbitrary area-preserving maps each KAM curve $\gamma$ can actually be constructed as a limit set of an appropriate family of $m$-periodic discrete invariant sets $\{I(m, n), m=m(n), n \in N\}$, i.e., for $n \rightarrow \infty$ :

$$
I(m(n), n) \rightarrow \gamma,
$$




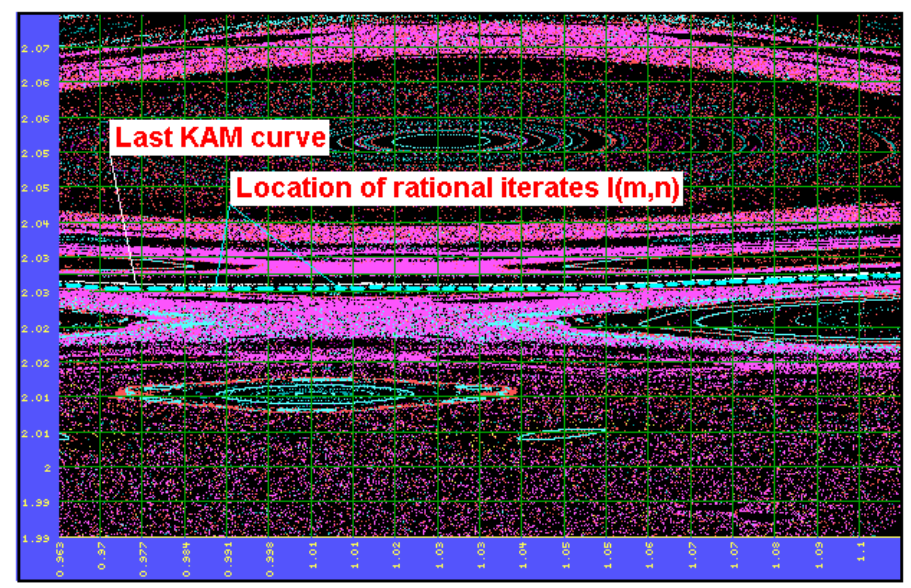

FIGURE 1. Location of last KAM curve and of the rational iterates

where the discrete invariant sets $I(m(n), n)$ are characterized by the distinctive property that their winding numbers $\alpha(I(m, n))=\frac{m(n)}{n}$ tend to $\alpha(\gamma)$ in the asymptotic limit, i.e.,

$$
\lim _{n \rightarrow \infty} \alpha(I(m, n))=\lim _{n \rightarrow \infty} \frac{m(n)}{n}=\alpha(\gamma) .
$$

A basic conjecture proposed by Greene [2]) is that for arbitrary area-preserving maps each KAM curve $\gamma$, having winding number $\alpha(\gamma)$, can actually be constructed as a limit set of an appropriate family of m-periodic discrete invariant sets (to be denoted as rational iterates) $\{I(m, n), m=m(n), n \in N\}$, i.e., for $n \rightarrow \infty$ :

$$
I(m(n), n) \rightarrow \gamma,
$$

where the discrete invariant sets $J(m(n), n)$ are characterized by the distinctive property that their winding numbers $\alpha(I(m, n))=\frac{m(n)}{n}$ tend to $\alpha(\gamma)$ in the asymptotic limit, i.e.,

$$
\lim _{n \rightarrow \infty} \alpha(I(m, n))=\lim _{n \rightarrow \infty} \frac{m(n)}{n}=\alpha(\gamma)
$$

\section{GREENE'S CHAOTIC HYPOTHESIS}

The method developed by Greene [2] for investigating stochastic transitions in area-preserving maps (and in particular for the standard map) is based on the following set of conjectures, denoted as Chaotic Hypothesis:

A) 1 st conjecture (irrational winding number of the last KAM curve): the last KAM surface is characterized by the irrational winding number

$$
\alpha(\gamma)=\alpha_{G M} \equiv \frac{\sqrt{5}-1}{2}
$$

$\left(\alpha_{G M} \equiv\right.$ golden mean) which can be represented by the continuous fraction

$$
\frac{\sqrt{5}-1}{2}=\frac{1}{1+\frac{1}{1+\frac{1}{1+. .}}} .
$$

B) 2nd conjecture (mechanism of destruction of a KAM curve) : the disappearance of a KAM surface (in particular the "last KAM surface" whose destruction produces the global stochastic transition) is related to the linear destabilization of the invariant points (elliptic equilibrium points) of an appropriate family of m-periodic discrete invariant 
sets $I(m, n)$, i.e. when the Lyapunov exponents of the invariant points become positive for suitable values of the stochasticity parameter $K$. The stochastic transition can be estimated by analyzing the bifurcation conditions (linear destabilization) of the invariant sets $I(m, n)$ for sufficiently high values of the order $n$ of these sets. In particular, for sufficiently high values of $n$ and for all $n_{1} \geq n$ all points of the sets $I\left(m\left(n_{1}\right), n_{1}\right)$ become linearly unstable.

C) 3rd conjecture (invariant sets): in the case of the last KAM surface of the standard map the discrete invariant sets $J(m, n)$ coincide with the so called rational iterates. The winding numbers of these special invariant sets are given by the following sequence, obtained truncating the previous continuous fraction, i.e.,

$$
\alpha(I(1,2))=\frac{1}{1+1}=\frac{1}{2}, \ldots, \alpha(I(m, n))=\frac{m(n)}{n}, \alpha(I(n, n+m))=\frac{n}{n+m}, \ldots \rightarrow \alpha_{G M},
$$

yielding the family of sets

$$
\{I(m(n), n), n \in N\} \equiv\{I(1,2), I(2,3), I(3,5), \ldots, I(m(n), n), \ldots\}
$$

where $n, m$ are Fibonacci numbers belonging to the sequence

$$
n, m \in\{2,3,5,8,13, \ldots k\},
$$

with $k$ given by the sum of the previous two elements of the sequence. Here $\mathrm{m}$ and $\mathrm{n}$ denote, respectively, the periodicity and the order (number of points) of the generic set of the family $I(m, n)$.

Using numerical experiments Greene was able to justify at least in part there conjectures and achieve the following results for the standard map:

I) identification of the last KAM curve and the family of rational iterates: (see figure 2) $\{I(m, n), n \in N\}$ (see figure 5): near stochastic transition the invariant sets $I(m, n)$ are found to be localized below the last KAM; these sets continue to exist also after transition (i.e., for values of the stochasticity parameter $K$ larger than the critical value $\left.K_{\text {crit }}\right)$.

II) linear stability analysis of rational iterates $I(m, n)$ : highest order $n$ considered for the rational iterate $n=233$, i.e., $I(m, n)=I(144,233)$

III) estimate of the critical value of the stochasticity parameter $K$ at stochastic transition:

$$
K \cong 0.9716 \text {, }
$$

determined by the requirement that the elliptic invariant points of the set $I(144,233)$ become linearly unstable.

Although these results have been confirmed by other authors using different approaches (Percival[3], Escande and Doveil[4] and MacKay[5]), they leave unexplained several aspects and basic issues of the theory. In particular, the identification of the invariant sets $I(m, n)$ (conjecture B) associated to the last KAM curve remains not obvious even for the standard map. In the same way this problem remains unanswered for arbitrary KAM curves and more general area-preserving maps.

\section{THE NONLINEAR CHAOTIC HYPOTHESIS (NCH)}

In this regard, a natural question is whether the family of invariant sets $I(m, n)$ (the rational iterates), associated to a given KAM curve, i.e., for which the KAM curve is a limit set, is unique or not. This issue arises immediately when trying to relate Greene's chaotic hypothesis to the well known Chirikov criterion for the overlapping of resonances (Chirikov Criterion [1]). In fact, the latter states that KAM curves are destroyed when the distance between the two lowest order resonances which encompass the same KAM curve, i.e., lie on opposite sides of the KAM curve, becomes comparable to the sum of the semi-amplitudes of the separatrix islands formed by the two resonances. On the other hand the existence of multiple sequences of discrete invariants sets having the same limit set (a given KAM curve) does not contradict the original Greene conjecture, which does not specify the precise location of the invariant sets $J(m, n)$ nor exclude their possible non-uniqueness. This has motivated us to search for possible candidates for these invariant sets, associated in principle to an arbitrary KAM curve, which are denoted here as alternate rational iterates $Y\left(m_{j}, n\right)$. Numerical experiments performed for the stochastic transition of the last KAM curve of the standard map [6] confirm this conjecture and suggest a new formulation of the Greene's Chaotic Hypothesis, replacing conjectures $\mathrm{B}$ and $\mathrm{C}$ with the following three new conjectures $\mathrm{B} 1$ and $\mathrm{C} 1$ :

B1) new 2nd conjecture (invariant sets related to a KAM curve) : the disappearance of a KAM surface $\gamma$ (in particular 
the "last KAM surface" which determines with its destruction the global stochastic transition) is related to the existence to two families of discrete invariant sets $I(m, n)$ and $Y\left(m_{j}, n\right)$, with $j$ a suitable integer, defined in such a way their winding numbers tend to $\alpha(\gamma)$, i.e.,

$$
\begin{gathered}
\lim _{n \rightarrow \infty} \alpha(I(m, n))=\lim _{n \rightarrow \infty} \frac{m(n)}{n}=\alpha(\gamma), \\
\lim _{n \rightarrow \infty} \alpha\left(Y\left(m_{j}, n\right)\right)=\lim _{n \rightarrow \infty} \frac{m_{k}(n)}{n}=\alpha(\gamma) .
\end{gathered}
$$

C1) new 3rd conjecture (invariant sets related to the last KAM curve of the standard map) : in the case of the last KAM surface of the standard map the discrete invariant sets $I(m, n)$ and $Y\left(m_{j}, n\right)$, coincide, respectively, with the sets of rational and alternate rational iterates when letting $j=1$.

D1) new 4th conjecture (nonlinear mechanism of destruction of a KAM curve): the stochastic transition of the last KAM surface occurs when the Euclidean distance between corresponding elliptic points of the sets $I(m, n)$ and $Y\left(m_{j}, n\right)$, considered as a function of the stochasticity $K$, becomes minima, for sufficiently high values of $n$.

The set of hypotheses A (Greene's first conjecture), together with B1,C1 and D1, is here denoted here as Nonlinear Chaotic Hypothesis (NCH). We stress that the conjecture D1 (new 4th conjecture) appears consistent with (but differs from) Chirikov Criterion, since the distance between nearby resonances cannot exceed the sum of the semi-amplitudes of the separatrix islands formed by the two resonances. Moreover, it replaces the assumption on linear stability with a non-linear destabilization criterion, i.e. the requirement that or the Euclidean distance between adjacent elliptic points belonging respectively to two sets $I(m, n)$ and $Y\left(m_{1}, n\right)$ having the same order $n$. In fact, in principle it is not obvious at all why a linear stability criterion (as Greene's 4th conjecture) should hold in general since an elliptic invariant point might result at the same time linearly unstably and nonlinearly stable. In addition, invariant points of different invariants sets (for example adjacent points invariants sets of the same order $n, I(m, n)$ and $Y\left(m_{1}, n\right)$ ), may not become all linearly unstable simultaneously, i.e. for the same value of the stochasticity parameter $K$.

Therefore, NCH should permit, in particular, not only a more accurate evaluation of the critical value of the stochasticity parameter $K$ occurring at stochastic transition, but also a precise identification of the families of discrete invariant sets associated, in principle, to an arbitrary KAM curve. Finally, the new approach closes the gap between Greene's Chaotic Hypothesis and Chirikov criterion of overlapping of resonances, permitting at the same time a deeper understanding of the mechanism of stochastic transitions in area-preserving maps.

\section{ACKNOWLEDGMENTS}

Work developed in the framework of the PRIN Research Program "Programma Cofin 2002: Metodi matematici delle teorie cinetiche"( MIUR Italian Ministry) and partially supported (for P.N.) by the National Group of Mathematical Physics of INdAM (Istituto Nazionale di Alta Matematica), (P.N) by the INFN (Istituto Nazionale di Fisica Nucleare), Trieste (Italy) and (M.T.) by the Consortium for Magnetofluid Dynamics, University of Trieste, Italy.

\section{REFERENCES}

1. B.V.Chirikov, Phys. Reports 52, 262 (1979).

2. J. Greene, J.Math.Physics 20, 1183 (1979).

3. I.C. Percival, in Nonlinear Dynamics and the beam-beam interaction, by M.Month and J.C.Herrera Eds., AIP Conference Proceedings No.67, American Institute of Physics, New York, 1979)

4. D.C.Escande and F.Doveil, Phys.Lett 83A, 307 (1981), Phys.Lett. 84A, 399 (1981) and J.Stat.Physics 26, 257 (1981).

5. R.S.MacKay, Renormalization in area preserving maps, Ph.D. Thesis Princeton 1982, World Scientific (1993).

6. M.Tessarotto and P.Nicolini, to be submitted (2004). 